usually intense, and that the livers and spleens of all those who died were found enlarged and congested. Kircksig, describing the epidemic of jaundice in Suden Scheid in 1794 , says that it raged from the end of August to the end of November; that the months of June, July and first half of August were characterized by prolonged heat, and dryness, followed by a sudden change of temperature and fall of the thermometer about the middle of August - the appearance of the epidemic commencing co-incidently with this decline of temperature. Innumerable instances of a similar nature, showing the direct connection of protracted heat with the existence of jaundice, might be cited, accomplishing such results no doubt by certain reflex actions transmitted from the sensitive surfaces through the cerebro-spinal and sympathetic systems to the involuntary muscles and secreting organs. The hepatic congestion and cholæmia found to exist in women during the catamenial presence, disappearing and returning cotemporaneously with the menstrual flow, furnishes another illustration of the effects upon the liver of reflex nervous excitation originating in the nerves of distant parts. Assuming then that the molecular processes going on in the protoplasm of the hepatic cells, necessary to the formation of bile pigment or the transformation of hæmoglobin into bllirubin, can be morbidly influenced by a disturbed condition of other organs through nervous connections, and that external causes - such as heat and cold-are capable of exerting such a power through the nerves of the integument, we are met by the question, in what manner does the deleterious agent of heat operate upon those nerves which control the function of the liver, to effect such derangement of its normal office?

Scientific research and experimentation have not yet supplied us with positive data upon which we can base a conclusive reply to this question. We can only fall back on the statements already made, and resting upon repeated experiments which demonstrate the effects upon the vascularity of the abdominal organs, including the liver, resulting from a division or a lesion of certain branches of the sympathetic nerve and by a legitumate method of logical deduction assert our belief in the theory that those branches of the sympathetic supplying the vessels of the liver, and influencing directly and potentially its office of secreting bile, when subjected to the protracted excitation and subsequent exhaustion of protecting the animal economy from the deleterious effects of prolonged heat, become partially paralyzed and are no longer capable of preserving through vaso-motor influence the normal arterial tonicity of the hepatic vessels; that this paresis of the arterial coats necessarily diminishes blood pressure and correspondingly increases venous congestion with a diffusion of bile into the circulation, and a consequent condition of jaundice; that such a result may not immediately follow the exposure to heat, but does in many instances develop itself by gradual morphotic changes going on and manifested at some subsequent period.

\section{AMPUTATION BELOW THE KNEE-JOINT IN PREF= ERENCE TO BRISMENT FORCE IN CERTAIN CASES OF DEFORMITY WITH AN = CHYLOSIS, ILLUSTRATED BY TWO CASES.}

BY LEWIS HALL SAYRE, M.D., ASSISTANT TO THE CHAIR OF ORTHOPEDIC SURGERY AT THE BELLEVUE HOSPITAL MEDICAL COLLEGE.

[Read in the Section on Surgery and Anatomy, June, 1883.] GeNTLEMEN :

In all chronic inflammations of the knee-joint, such as strumous synovitis, white swelling, fungus articuli, etc., there is a reflex muscular contraction, which, unless prevented or overcome by persistent extension and counter-extension during the progress of the disease, will result in more or less serious deformity, generally a partial or incomplete sub-luxation backward with outward rotation, in which position it may be anchylosed by fibrous adhesions, false anchylosis, or by bony fusion, true anchylosis.

In all cases where the disease has entirely subsided, leaving this deformity, if it is possible to separate the patella from its adhesions with the femur, and if there is any movement whatever between the tibia and the femur, it is easier to resort to brisment forcé, even if it be necessary to make subcutaneous resection of the hamstring tendons in order that the leg may be brought into proper position. This operation, followed by the proper after-treatment, frequently results in a useful limb, and not infrequently with a movable joint.

But $i_{i 1}$ cases where the patella is absolutely immovable axd the tibia and femur aré united by long fusion, it becomes necessary to make a $\mathrm{V}$ section through the angle of deformity as suggested by the late Dr. Gordon Buck, of New York, straighten the limb and secure it in that position by anchylosis. In all cases where the limb is of sufficient length to make it useful for locomotion without too great shortening, this is the preferable treatment as it results in a very useful member.

In those cases where the disease of the joint has occurred at a very early period of life, and has resulted in this deformity, the limb below the knee grows much less rapidly than the other. Patients are frequently brought to you in early adult life with the limb very much shorter than its fellow, and by the time they reach maturity the difference in the length of the limbs would make so serious a deformity that an artificial limb would be preferable to the natural one in its shortened condition. In all such cases, amputation below the knee-joint in the manner which I here intend to propose, and which is a modification of Prof. Stephen Smith's amputation at the knee-joint is preferable to resection of the bone, and attended with very much less danger to the patient.

The amputation should be performed by passing the $\mathrm{k}$ ife from the tubercle of the tibia slightly downward and backward to the popliteal space, making a very slightly curved flap; then passing the knife from the same point on the tibia around the other side of the leg to the popliteal space with a similar curve, meeting your first incision at that point. 
These flaps are then dissected up from a half an inch to an inch on either side, according to size of the limb; a free incision is then made throngh the legamentum patellæ at its insertion into the tubercle of the tibia and the remaining soft tissues down to the bone. Then separate the tibia just below its articular facets either by a strong cartilage knife, or if the bony fusion is complete it is preferable to use the saw, in this way the joint is not exposed, and if the Esmarch bandage be used the operation is comparatively bloodless.

The popliteal artery being now secured, the thigh is flexed at a right angle with the trunk; the wound is then stitched in the posterior part, making a linear incision entirely posterior to the end of the stump, a drainage tube having been inserted making its exit at the posterior portion of the wound at the upper part of the thigh.

If this operation be performed with antiseptic precautions, union, by first intention, can as a rule be secured, and no constitutional disturbance results, and when the wound is healed, the end of the stump, being free from cicatricial tissue, is never subject to irritation by pressure from an artificial limb.

The advantages of this operation are :

I. Much less dangerous than resection of the bone, resulting in much more speedy cure.

2. Resection always results in anchylosis, which prevents flexion of the limb in the sitting posture, which is a very serious inconvenience to the patient, while an artificial limb can be so applied as to be flexed when sitting, and is equally serviceable in locomotion.

3. If the natural limb is so much shortened as to require elongation by an artificial foot, or a very high shoe, the support is very insecure in walking, while the deformity is much more conspicuous than an artificial limb.

4. The advantage of this amputation over those ordinarily used is that the end of the stump is able to bear the weight of the patient without danger of irritation, the cicatrix being entirely behind the limb, and therefore not subjected to any pressure.

Eva L. J., aged I6; father healthy, mother always delicate, as has been this child; seven other children all healthy. When patient was 18 months old an abscess formed at the top of the left knee, which opened at the lower part of the joint. No cause was known for this. The inflammation of the joint continued until she was between 5 and 6 years old, when the leg began to be flexed upon the thigh. One night the limb became flexed at nearly a right angle, in which position it has since remained. There was considerable tenderness of the joint, which was treated by liniments, frictions, etc. No extension was ever used. The patient at present is in fair health. Left limb flexed at nearly a right angle as seen in drawing, Fig. I, subluxated barkward and everted, firmly anchylosed by fusion of the femur, tibia, and patella. The leg below the knee is more than 3 inches shorter than its fellow. For this reason amputation below the knee was decided upon, as a $\mathrm{V}$ section through the angle of deformity would result in so great a shortening as to render the limb useless for locomotion without an elongated shoe, and, being at the same time anchylosed, would not have the advantage of flexion, which an artificial limb would give.

The modification of Smith's amputation was therefore performed by Dr. Lewis A. Sayre on April 7, 1883, with antiseptic precautions. There were no untoward symptoms, the ligatures coming away on the eleventh day, and the wound being completely closed on the seventeenth day after the operation, so that an artificial limb was applied six weeks from day of operation on which she walked without pain or discomfort. The accompanying photographs, taken one week after the application of the limb, show the condition of the stump and the position of the cicatrix entireiy posterior to its end, as well as the ability to stand upon the limb, also that of flexion at the knee while sitting.

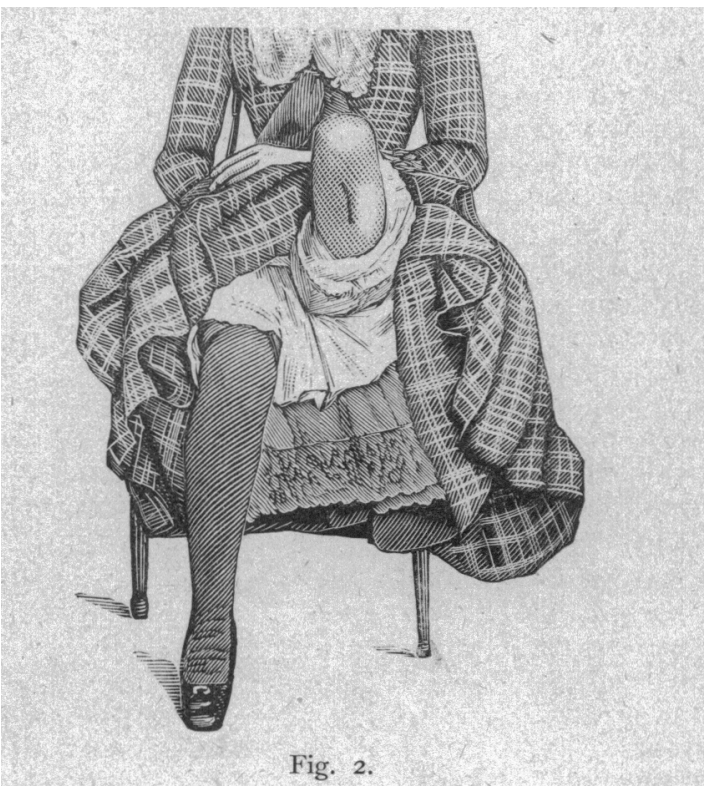




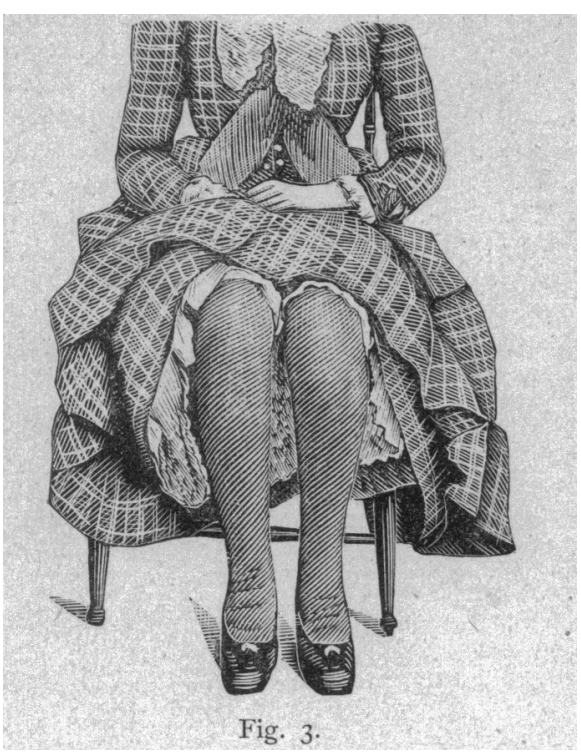

E. G., aged II, of healthy parents, and always perfectly healthy. When 4 years of age, mother noticed swelling of right knee. This was pronounced white-swelling by the physician, who treated it by blisters and fixation in a box-splint, no extension being used. The limb was retained in the splint for four months, when it was removed, and the knee found to be anchylosed in the straight position. This anchylosis was fibrous in its character, as during the next four or five years the knee became more and more flexed, until it is now fixed at a right angle, with displacement of the tibia backward.

There is slight motion between the tibia and femur, but the patella is firmly fixed. An attempt at brisment forcé was made on April 25, I883, but it was found impossible to release the patella from its fastenings to the femur, and the operation was therefore abandoned. No trouble followed the attempt at brisment forcé. The limb has been kept immovable after the operation, with pressure on the femoral artery, and cold applications to the joint, and on May 23, I883, I amputated the limb below the knee-joint in the manner described, separating the tibia at the cartilaginous junction.

A small abscess was discovered in the head of the tibia, just beneath the cartilage. This was thoroughly scraped, and the wound washed with carbolized water. A ligature was placed upon the popliteal artery, a drainage tube inserted, and the wound closed with five stitches and adhesive-plaster strips between them. The operation was performed antiseptically. No trouble has followed, the temperature and pulse having not risen above roo, and very slight pain having been experienced.

Forty-eight hours after the operation the limb was dressed under the spray. No pus found. The adhesive straps were left in situ, but the sutures carefully removed without disturbing the wound, which seemed to be united except at the point of exit of the drainage tube and ligature, when the limb was re-dressed as before.

\section{PAEDIATRIC MEDICINE AND ITS RELATION TO} GENERAL MEDICINE.

BY J. B. CASEBEER, M.D., AUBURN, IND.

[Read in the Section on Diseases of Children, June, r883.]

Many of our most successful practitioners of medicine amongst the adult population have made signal failures when called upon to exhibit their skıll in the treatment of tender children.

We have often been pained by the remarks dropped from the lips of some physicians whom we were endeavoring to regard as sample practitioners, on account of the indifference manifested, and the slight degree of importance attached to their practice among the children, such as, "Well, you may give a few drops of 'paregoric,' or some ' catnip tea,' or most anything of that kind you may find convenient, as we cannot do much for children so young;" or, "Your mothers or 'old women' can treat young children as well as I or any physician can ;" or, "I don't like to treat children, it is so unsatisfactory. They cannot tell how they feel and what is the matter with them, and I never can tell what they need."

To my mind, such remarks (and these are but a few imperfect specimens of those somewhat current in our ranks) indicate a lack of knowledge, or a misconception of the true principles of the practice of medicine as applied to the children, which certainly challenges our sincere attention.

It is a loud cry for reform or re-education. To my mind, the diseases of children speak as plain a langnage, and require as direct remedies to control them, and as emphatic in their demands as are those of the adult; and when we admit that untutored women or good nurses can judge of the requirements of sick children, and can treat them as well as the educated physician, then I feel that we ought to be honest enough to admit that the same is true in all classes of the sick. To my mind, paregoric, rhubarb, and chamomile are appropriate remedies, when indicated, alike to the child and the adult, and when not indicated would be as much out of place in the one as the other.

The natural language of the child is the true and untarnished expression of facts; while that of the adult, tainted by the prejudice of their own opinions, freighted with the imperfect use of an imperfect language to express their imperfect thoughts, must be taken with a great deal of allowance, and very much of it must be often entirely excluded when making up a diagnosis.

If the intelligent physician will but open his eyes and his ears, his little patient will unconsciously tell him how he feels and what is the deviation from the health standard. If he will but study cause and effect, he will find the indications for treatment just as plainly marked out as in his older patient, that may be very fluent in reciting his pains and aches. The physician practicing rational medicine, and who is governed in his treatment by the indications of each individual case, and strives to meet only the indications that do exist, needs to be versed in the laws and natural language of disease, the laws and standard of health, the true nature and effect of the reme- 